\title{
Resonance Capture and Saturn's Rings
}

Chris W. Patterson

$$
\begin{aligned}
& \text { LA-- } 10727-M S \\
& \text { DE86 }
\end{aligned}
$$




\title{
RESONANCE CAPTURE AND SATURN'S RINGS
}

\author{
by
}

Chris W. Patterson

\begin{abstract}
ABS IRA.CT
We have assigned the resonances apparently responsible for the stabilization of the Saturn's shepherd satellites and for the substructure seen in the F-ring and the ringlets in the C-ring. We show that Saturn's narrow ringlets have a substructure determined by three-body resonances with Saturr's ringmoons and the Sun. We believe such resonances have important implications to satellite formation.
\end{abstract}

In a previous paper (Patterson 1985), we have shown that the outer planets are presently close to two-body resonances whereas the inner minor planets or asteroids are close to three-body resonances. We attribute this to the formation of planets as a result of resonance capture of planetesimals by the initial binary system of the Sun and Jupiter. The rules for preventing orbital decay of planetesimals by resonance capture are consistent with these planetary observations: outer planetesimals require at least two-body resonances for capture whereas inner planetesimals require at least three-body resonances for capture in a gas-drag environment.

We might expect similar resonances to govern the formation of planetary satellites and rings. This hypothesis provided the motivation for a systematic search for possible two- and three-body resonances of Saturn's satellites and rings. Such a search has been undertaken before by Borderies et al. (1984) in an attempt to explain the extremely short time scale for satellite orbital evolution near the rings. In particular, according to Borderies et al., the shepherd satellites Pandora (1980S26) and Prometheus (1980S27) could have moved from the A-ring boundary to their present location in approximately 20 and $4 \mathrm{Myr}$, respectively. Unless these satellites 
were stopped by some orbital resonances with outer satellites, they appear to have originated relatively recently. A similarly short time scale occurs for the evolution of the coorbital satellites Janus and Epimetheus. According to Lissauer et al. (1985), these satellites will evolve from a 'horseshoe' orbit to a 'tadpole' orbit in approximately $20 \mathrm{Myr}$. We show below evidence for two-body satellite resonances that can lengthen the above time scales appreciably.

We first look for the nearest two-body interior Lindblad resonances involving Saturn's ringmoons and ringlets. We follow the procedure of Lissauer and Cuzzi (1982) who have calculated the strong two-body Lindblad resonances of Saturn's ringmoons. Many of Saturn's ringlets have already been assigned to these resonances by Holberg et al. (1982). Let $\Omega$ corre-spond to a ring particle's mean motion and $\omega$ to the motion of its apsidal precession. Then the $\ell / m$ inner Lindblad resonance with a satellite satisfies the condition

$$
\ell \Omega=m \Omega_{s}-\omega-(m-\ell-1) \omega_{s} .
$$

where $\Omega_{S}$ and $\omega_{S}$ are the satellite's mean motion and precessional motion, respectively. Our notation is consistent with our previous work, but we caution the reader that the above resonance is of ten labeled $\mathrm{m} / \ell$.

Table I represents a modest extension of the results of Lissauer and Cuzzi to include higher order resonances with up to power $(m-\ell-1)=2$ in the satellite's eccentricity, $\epsilon_{S}$. The accuracy of these calculations should be of the order of $10 \mathrm{~km}$. The ringlets of Saturn and the shepherd satellites in Table I occur near very weak two-body Lindblad resonances.

Torque exerted by the A-ring on the shepherd satellites causes them to move outward, making two-body resonance capture possible. As seen in Table I, the shepherd satellites Pandora and Prometheus occur within the expected 10-km error of the calculated two-body resonances with Epimetheus. The evidence for these resonances is rather convincing because the spacing between resonances of this order is $\sim 500 \mathrm{~km}$. Both of these resonances are of first order in Epimetheus eccentricity. We would expect that Atlas, the ringmoon nearest to the rings, would be captured by a $j / j+1$ resonance with 
TABLE I

RESONANCES OF SATURN'S SHEPHERD SATEILITES AND RINGLETS

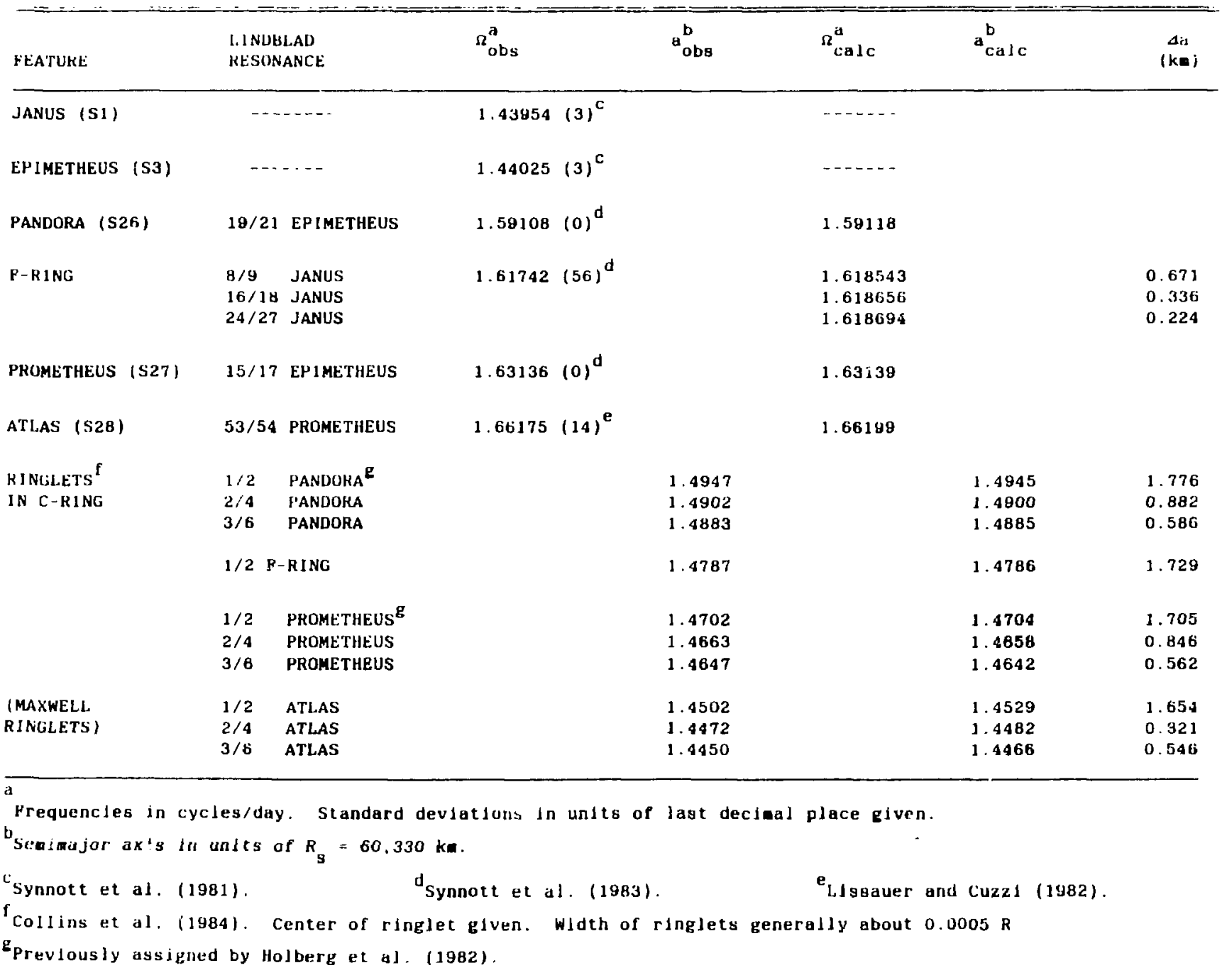


Prometheus, the satellite nearest to $1 \mathrm{t}$. The spacing of resonances of this order near Atlas is only $\sim 30 \mathrm{~km}$, however, so that the experimental and calculational errors make assignment difficult. Because of these resonances, the coorbital satellites exert a negative torque on the shepherd satellites. slowing their orbital evolution. The work of Lissauer et al. (1985), which only considers the differential ring torques on the coorbiting satellites, must now be reevaluated. He could not find any similar low-order resonances that would capture the coorbital satellites. However, the case is certainly strengthened for a more ancient origin of the shepherd satellites and Atlas.

The torque exerted by the shepherd satellites on the rings causes them to move inward, making three-body resonance capture a possibie mechanism to stabilize their trajectories. Saturn's ringlets provide evidence for such ongoing three-body resonances. These ringlets are known to have a dense substructure, which has been seen in both star occultations (Lane et al. 1982 and Esposito et al. 1984) and radio occultations (Cuzzi et al. 1984). For example. we show in Fig.1 the Voyager 2 photopolarimetry spectrum of the F-ring taken by Lane et al. (1982). Figure la shows the bands of the F-ring at $\approx 1 \mathrm{~km}$ resolution, and Fig. $1 \mathrm{~b}$ shows the fine structure subbands of a portion of this spectrum at $\approx 100 \mathrm{~m}$ resolution.

The orbital frequency of the F-ring center was measured by Synnott et al. (1983) and is shown in Table I. From Table I, we find that the $8 / 9$ inner Lindblad resonance of Janus is some $65 \mathrm{~km}$ inside the observed F-ring center. In Fig. 1a, we place this resonance at the origin some $45 \mathrm{~km}$ from the F-ring 'band head.' which is well within the experimental and calculational errors of Table I. From Table I, we find that the $16 / 18$ and $24 / 27$ Lindblad resonances of Janus are inwardly displaced by 6.52 and $8.69 \mathrm{~km}$ from the $8 / 9$ resonance, as shown by the dotted lines in Fig. 1a.

It is interesting that there appears to be a small gap at the $8 / 9$ resonance of Janus in the F-ring. Gaps in the Cassini division corresponding to even weaker resonances have been found by Wiesel (1982). He may readily estimate the expected width of this gap if we assume it is equal to the width of the resonance zone for librational orbits given by Franklin et al. (1984) or $\delta a \approx a\left(m_{J}\right)^{2 / 3}$, where $m_{J}=8 \times 10^{-9}$ is the mass of Janus relative to the mass of Saturn. We find $\delta \approx 1 \mathrm{~km}$, which is of the order of the observed gap.

The coorbiting satellites are known to change their orbital frequencies in horseshoe orbits approximately every 4 years (Yoder et al. 1983), and 
Saturn's F-ring

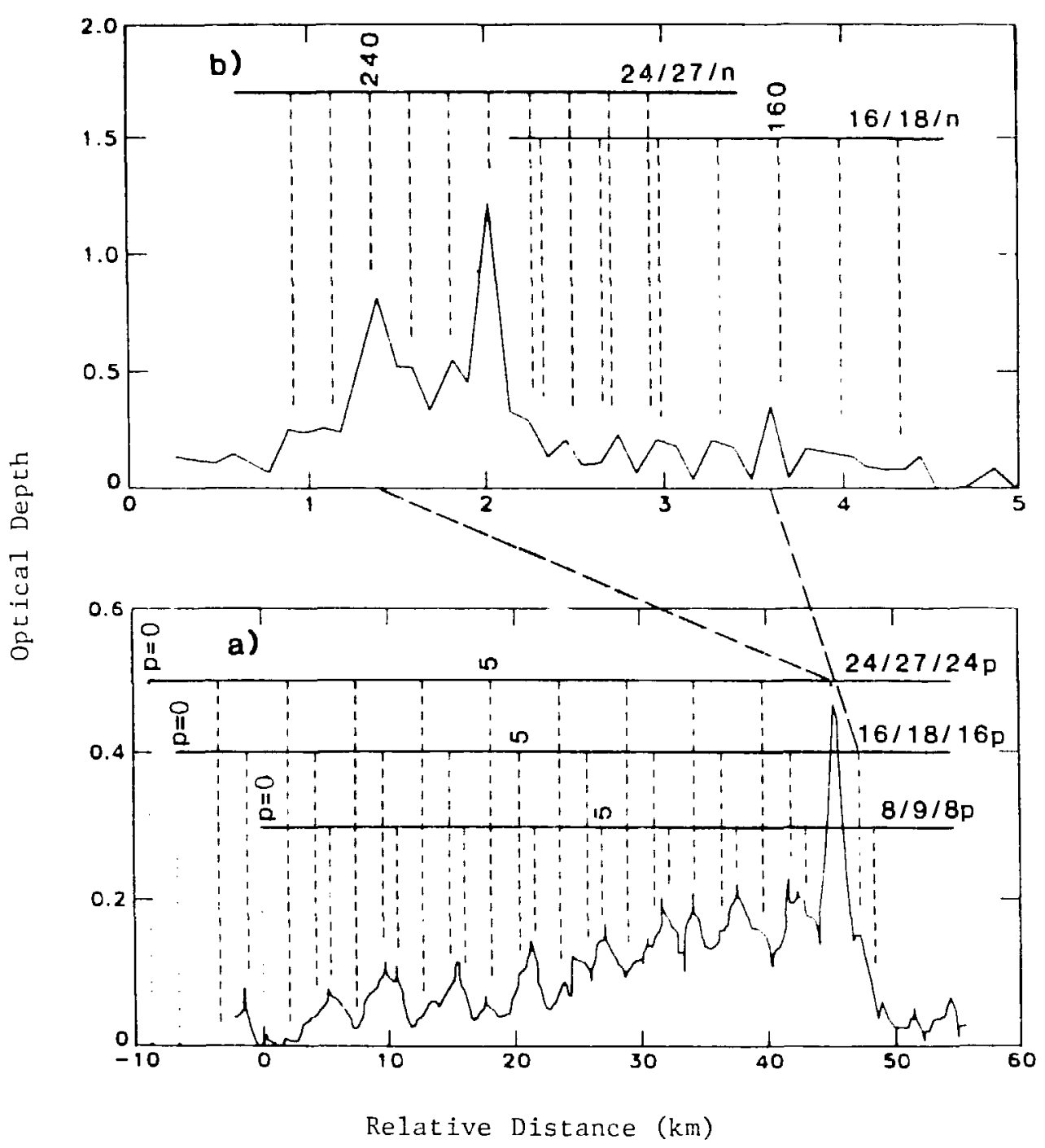

Fig. 1. Photopolarimetry of star occultation by F-ring after Lane et a1. (1982). (a) Spectrum with $\mathrm{l}-\mathrm{km}$ resolution. The dotted Jines show the positions of the two-body Lindblad resonances $\ell / m$, and the dashed lines show the positions of the three-body resonances $\ell / \mathrm{m} / \ell p$ with $\mathrm{p}=0$ to $\mathrm{p}=10$. The interval, $5.37 \mathrm{~km}$, between dashed lines of each of the three progresssions corresponds to the frequency interval of the Sun's orbit around Saturn. (b) Spectrum with 100-m resolution, showing the two dominant three-body progressions $\mathrm{k} / \mathrm{m} / \mathrm{n}$ at the band head in (a). 
the resonances attributed to these satellites in Table I occurred during the epoch of the Voyager 2 flyby in 1981. The next 4-year epoch began in 1982. The predicted position of the $8 / 9$ resonance of Epimetheus in Fig. la after Epimetheus' horseshoe alternation with Janus in 1982 would be displaced approximately $20 \mathrm{~km}$ outward from the $8 / 9$ resonance of Janus in 1981 . Thus, the experimental error of $30 \mathrm{~km}$ in the F-ring's position (as derived from its orbital frequency) makes it impossible to assign the $8 / 9$ resonance in Fig. la tc Janus or Epimetheus with certainty. The same ambiguity does not exist for the resonances with Pandora and Prometheus because their orbital frequencies are more accurately known. Because switching times are comparatively short, these resonances are operative during half of a coorbital satellite's S-year cycle in its horseshoe orbit.

Each of these two-body resonances serves as an origin for a progression of three-body resonances at intervals of the Sun's orbital frequency around Saturn, as shown with dashed lines in Fig. la. Other weaker progressions are possible, but these three acrount for most of the structure seen in Fig. 1a. For a $\ell / m$ Lindblad resonance, we may define the three-body resonances $\ell / m i n$ such that

$$
l \Omega=m \Omega s-\omega-(m-l-1) \omega_{s}+n \Omega_{S} \text { Sun },
$$

where $\Omega_{\text {Sun }}$ is the negative of Saturn's orbital frequenc's around the Sun. In the region near the F-ring, this frequency interval, $\Omega_{\text {Sun }}=-0.03395$ cycles/ year, corresponds to a radial interval of $+5.37 \mathrm{~km}$. We have ignored Saturn's small precessional frequency in Eq. (2). As seen in Fig. la, the three-body resonance appears to be strongest when $\mathrm{n}$ is an integer multiple of $\ell$, or $n=e_{p}$, corresponding to the dashed lines in Fig. 1a, with the periodicity of the Sun's orbital frequency.

The three progressions shown in Fig. 1a agree quite well with the peaks in the spectrum and account for the numerous bands seen in the F-ring. The strongest and lowest order progression 8/9/8p generally corresponds to the highest peaks whereas the weakest and highest order progresssion 24/27/24p corresponds to the lowest peaks. A notable exception is the band head at $24 / 27 / 24$ p with $p=i 0$ near $45 \mathrm{~km}$ in Fig. la. Within the errors of Table I, the 
only free parameter in the progressions shown in Fig. la is the origin of the two-body $8 / 9$ resonance. The origins of the other two progressions are fixed relative to this progression by Saturn's gravitational harmonics. The reason why the optical depth of the F-ring bands increases with $p$ and why the band head occurs at $45 \mathrm{~km}$ in Fig. la is unknown to us.

The band head at $45 \mathrm{~km}$ in Fig. $1 \mathrm{a}$ is expanded in Fig. 1b. The fine structure subbands of this spectrum are again explained in terms of the three-body resonance progressions. Near the band head at 24/27/24p with $p=10$, we see a progression $24 / 27 / n$ in Fig. $1 b$ with intervals of $\Omega_{\text {Sun }} / 24$. corresponding to $(5.37 / 24)=0.224 \mathrm{~km}$. Similarly, near the peak at $16 / 18 / 16 \mathrm{p}$ with $\mathrm{p}=10$, we see a progression $16 / 18 / \mathrm{n}$ in Fig. 1b with intervals of $\Omega_{\text {Sun }} / 16$. corresponding to $(5.37 / 16)=0.336 \mathrm{~km}$. We have chosen regions of the spectrum in Fig. lb where one progression dominates. In general, we will see substructure arising from an overlap of all three progressions. Note that the displacenent of the two progressions shown in Fig. $1 \mathrm{~b}$ is fixed by the relative displacements of their origins, namely, $2.17 \mathrm{~km}$, as in Fig. $1 \mathrm{a}$.

The fact that such weak resonances contribute to the F-ring bands and subbands helps to substantiate the assignments of other ringlets given in Table I. As in the case of the F-ring, the ringlets in Table I occur in triplets, except for the ringlet apparently generated by the F-ring itself. This latter ringlet is generated by a very weak resonance and consequently has no higher-order 'sidebands.'

Unlike the F-ring triplet, however, the C-ring triplets are well separated and should exhibit simple nonoverlapping subbands. The predicted spacing of these subbands, $\Delta a$, is given in the last column of Table I. We note that the $1 / 2$ Pandora $r i n g l e t$ is coincident with the $1 / 3$ Mimas ringlet (Holberg et al. 1982), resulting in a more complicated substructure. In principle, the substructure can occur in progressions emanating both outward and inward from the two-body gap at the origin; that is, $n$ in Eq.(2) can be both positive and negative. This could result in two band heads for each of the ringlets. Perhaps such behavior is not exhibited by the F-ring oring to the influence of the nearer and larger shepherd satellite, Prometheus.

Perhaps the occurrence of ringlets at three-body resonances explains some of their puzzling aspects, as described by Borderies et al. (1984). In particular, these authors noted difficulties with the shepherding mechanism confining the F-ring. The occurrence of three-body resonances explains the 
confinement of ringlets as well as their stabilization against orbital change--with the Sun providing the necessary energy. The occurrence of three-body resonances could also explain why the transport of material by spiral density waves was less then expected (Goldreich and Tremaine 1978). because this transport would be hindered by three-body resonance capture. Indeed, one must be careful not to confuse density waves with the apparently widespread occurrence of weak resonances, which can interact both constructively and destructively in the ring system. The rings will be especially difficult to decipher if resonances of eccentric rings can themselves lead to other rings as appears to be the case with the $1 / 2 \mathrm{~F}-\mathrm{ring}$ resonance at $\mathrm{a}=1.4787$ in Table $I$.

Because the ringlet particles are locked in phase with the Sun's orbit around Saturn owing to three-body resonances, there is no differential precession observed within the narrow ringlets of the C-ring. Apparently the strength of tre three-body resonances is sufficient to overcome any differential precession. This phase locking occurs separately for each of the independent three-body progressions arising from the different Lindblad resonances. This means that there can be differential precession between the different progressions shown in Fig. 1a. Such a differential precession between the three progressions in Fig. 1a would lead to the appearance of separate strands, as was seen in the Voyager 1 images (Dermott 1984). This differential precession is complicated and apparently is negligible in the region of Fig. 1. Indeed, like the complicated F-ring braiding, kinking, and knotting, it appears to be partly controlled by the shepherd satellites.

Finally, we comment on the possible role of resonant capture on satellite formation. If we assume that Saturn's largest satellite. Titan, was the first to form, then we might expect the satellites outside of Titan to form at two-body resonances and those inside Titan to form at three-body resonances owing to resonance capture of planetesimals in anology with previous planetary considerations (Patterson 1985). Yet, as shown by Greenberg (1984), the closest resonances of Saturn's satellites involve only two-body resonances.

We may resolve this dilemma if the "inner" satellites were initially rings at three-body resonances such that they were displaced from two-body resonances by frequency intervals of the Sun's orbit around Saturn. Because the frequency intervals of the Sun are exceedingly small, such three-body 
resonances would be indistinguishable from two-body resonances once the satellites accreted. For example, it is possible that the shepherd satellites initially accreted from rings at three-body resonances with the coorbital satellites and the F-ring is an unaccreted remnant. A similar scenario in which the coorbital satellites themselves accreted from a narrow ring has been proposed by Dermott (1984).

The rings of Saturn interior to Atlas cannot accrete into satellites because they are presumably inside the Roche limit. Thus, the rings might provide information on the initial stages of satellite formation, much like the asteroids shed light on intermediate planetary formation.

\section{ACKNOWLEDGMENT}

The author would like to thank Russell Kidman. David Hanson, and Jeffrey Hay for their continuing interest and support.

\section{REFERENCES}

N. Borderies, P. Goldreich, and S. Tremaine, "Unsolved problems in planetary ring dynamics," in Planetary Rings, R. Greenberg and A. Brahic, Eds. (Univ. of Arizona Press, Tucson, 1984), pp. 713-734.

S. A. Collins, J. Diner, G. W. Garneau, A. L. Lane, E. D. Miner, S. P. Synnott. R. J. Terrile, J. B. Holberg, B. A. Smith, and G. L. Tyler, "Atlas of Saturn's Rings," in Planetary Rings, R. Greenberg and A. Brahic, Eds. (Univ. of Arizona Press, Tucson, 1984), pp. 737-743.

J. N. Cuzzi, J. J. Lissauer, L. H. Esposito, J. B. Holberg, E. A. Marouf, G. L. Tyler, and A. Boischot, "Saturn's rings: properties and processes," in Planetary Rings, R. Greenberg and A. Brahic, Eds. (Univ. of Arizona Press, Tucson, 1984), pp. 73-199.

S. F. Dermott, "Dynamics of narrow rings," in Planetary Rings, R. Greenberg and A. Brahic, Eds. (Univ. of Arizona Press, Tucson, 1984), pp. 589-637.

L. W. Esposito, J. N. Cuzzi, J. B. Holberg, E. A. Marouf, G. L. Tyler, and C. C. Porco, "Saturn's rings: structure, dynamics, and particle properties," in Saturn, T. Gehrels and M. Matthews, Eds. (Univ. of Arizona Press, Tucson, 1984).pp. 463-545.

F. Franklin, M. Lecar, and W. Wiesel, "Ring particle dynamics in resonances," in Planetary Rings, R. Greenberg and A. Brahic, Eds. (Univ. of Arizona Press, Tucson, 1984), pp. 562-588. 
P. Goldreich and $S$. Tremaine, "The formation of the Cassini division in Saturn's rings," Icarus 34, 240-253 (1978).

R. Greenberg, "Orbital resonances among Saturn's satellites," in Saturn. T. Gehrels and M. Matthews, Eds. (Univ. of Arizona Press, Tucson, 1984), pp. 593-608.

J. B. Holberg. W. T. Forrester, and J. J. Lissauer, "Identification of resonance features within the rings of Saturn," Nature 297, 115-120 (1982).

A. L. Lane, C. W. Hord, R. A. Hest, L. W. Esposito, D. L. Coffeen, M. Sato, K. E. Simmons, R. B. Pomphrey, and R. B. Morris, "Photopolarimetry from Voyager 2: preliminary results on Saturn, Titan, and the rings," Science 215. $537-543$ (1982).

J. J. Lissauer and J. N. Cuzzi, "Resonances in Saturn's rings," Astron. J. 87, $1051-1058$ (1982).

J. J. Lissauer, P. Goldreich, and S. Tremaine, "Evolution of the Janus-Epimetheus coorbital resonance due to torques from Saturn's rings." Icarus $\underline{64}$, 425-434 (1985).

C. W. Patterson, "Resonance capture and the evolution of the planets," Los Alamos National Laboratory document LA-UR-85-4289.

S. P. Synnott, C. F. Peters, B. A. Smith, and L. A. Morabito, "Orbits of the small satellites of Saturn." Science 212, 191-192 (1981).

S. P. Synnott, R. J. Terrile, R. A. Jacobson, and B. A. Smith, "Orbits of Saturn's F ring and its shepherding satellites," Icarus $\underline{53}, 156-158$ (1983).

W. Wiese1, "Saturn's rings: resonance about an oblate planet," Icarus $\underline{51}$. 149-154 (1982).

C. F. Yoder, G. Colombo, S. P. Synnott, and K. A. Yoder, "Theory of motion of Saturn's coorbiting satellites," Icarus $\underline{53}, 431-443$ (1983). 


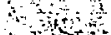

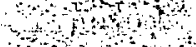

1t?

ntw

,

$+1$

tron.

Wh H.

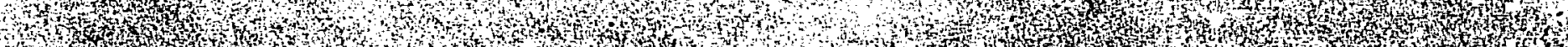

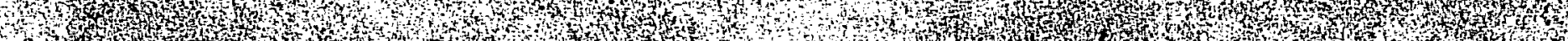

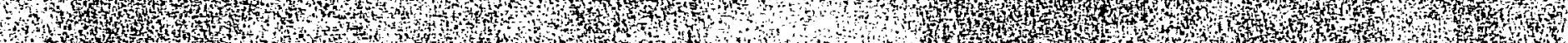

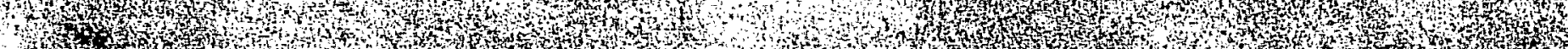
36.

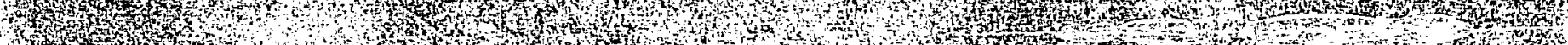

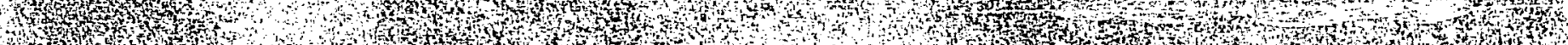

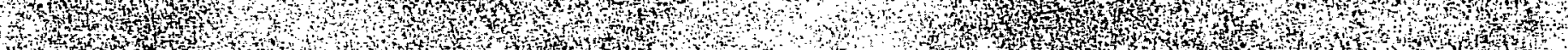

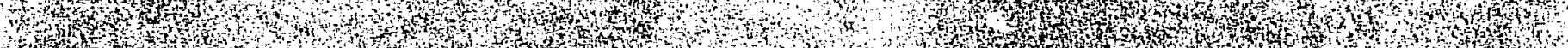
r

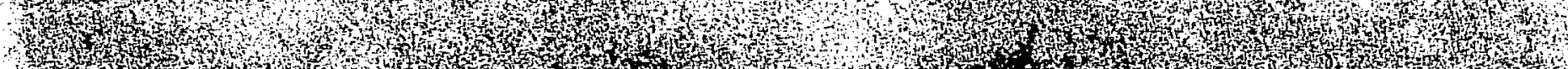

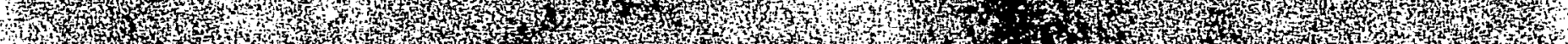
1. 4. 15. 19 1.1.

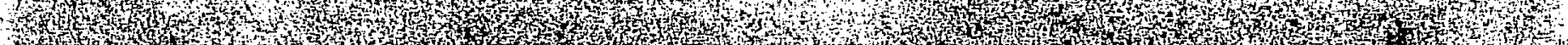

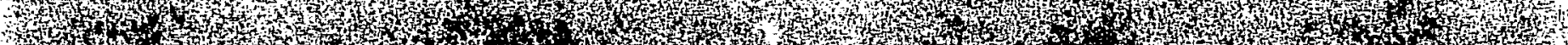

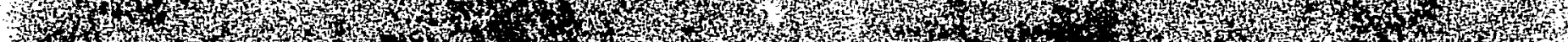
7.

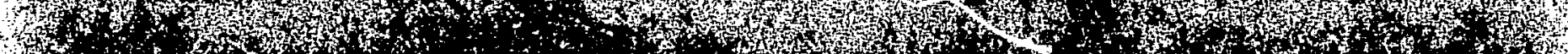

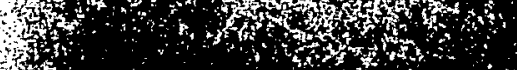

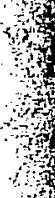

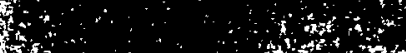

(1)

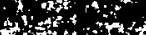

30

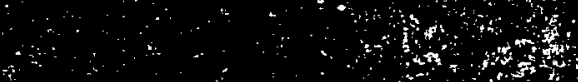

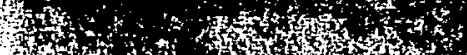

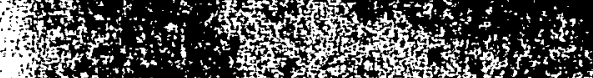

W6

ra

3.

\%

14

to

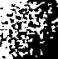

.

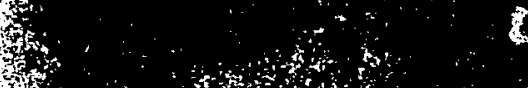

Hos

W $x^{2}+4+4$

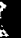

5

in

t.

in



th

ty

I

to

W

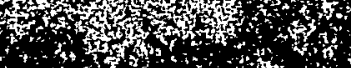

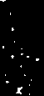

$\cdots$

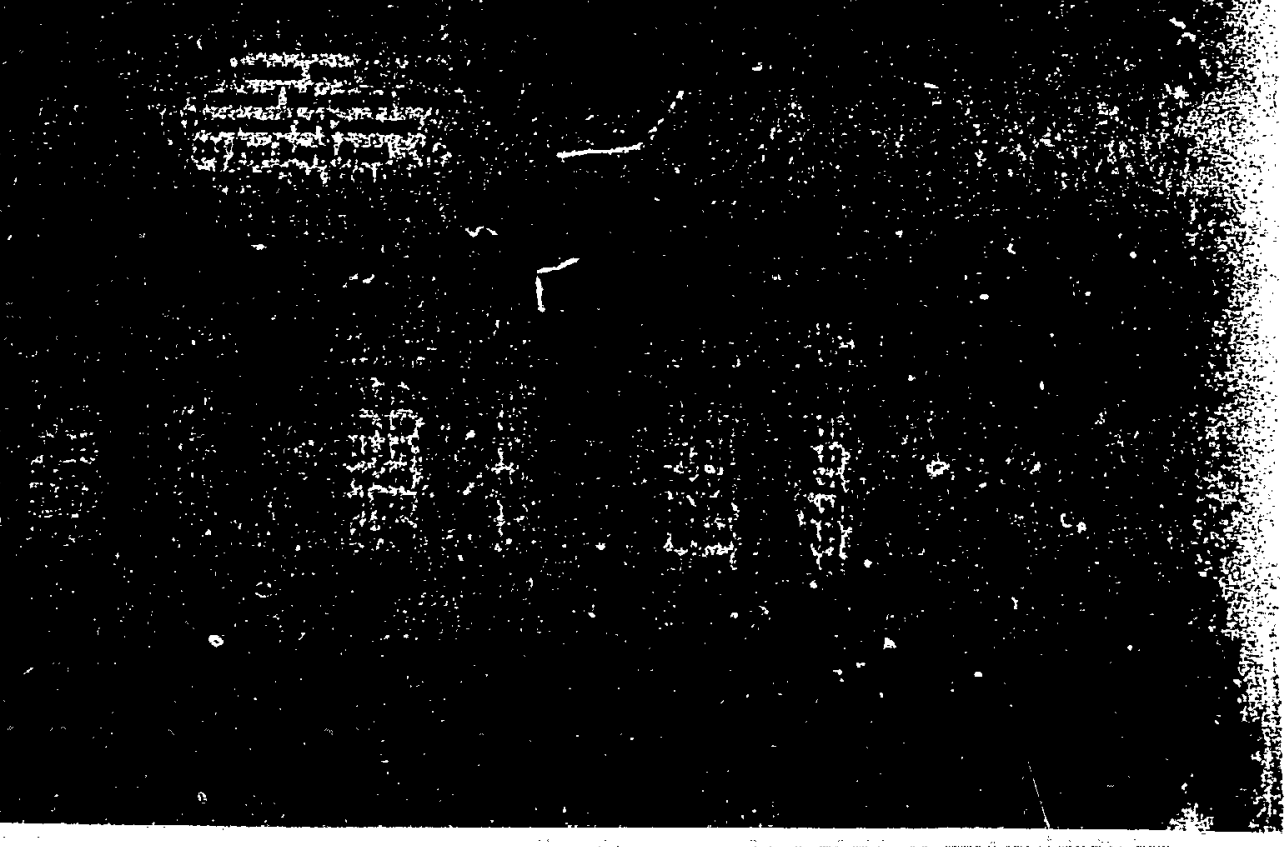

m, $x_{3}$

How

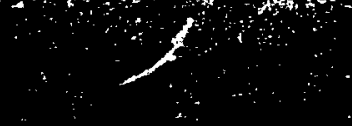

